\title{
NATURALLY ORDERED REGULAR SEMIGROUPS WITH MAXIMUM INVERSES
}

\author{
by TATSUHIKO SAITO
}

(Received 24th March 1987)

\section{Introduction}

Let $S$ be a regular semigroup. An inverse subsemigroup $S^{\circ}$ of $S$ is called an inverse transversal if $S^{\circ}$ contains a unique inverse of each element of $S$. An inverse transversal $S^{\circ}$ of $S$ is called multiplicative if $x^{\circ} x y y^{\circ}$ is an idempotent of $S^{\circ}$ for every $x, y \in S$, where $x^{\circ}$ denotes the unique inverse of $x \in S$ in $S^{\circ}$. In Section 1, we obtain a necessary and sufficient condition in order for inverse transversals to be multiplicative.

It is well known that the set $E(S)$ of idempotents of a regular semigroup $S$ can be partially ordered by setting $e \omega f \Leftrightarrow e f=f e=e$ for any $e, f \in E(S)$. This partial order is called the natural order on $E(S)$. A partially ordered semigroup $S(\cdot, \leqq)$ is called naturally ordered if the order $\leqq$ extends the natural order $\omega$ on $E(S)$, i.e. $e f=f e=e$ implies $e \leqq f$. In this case, there is no assumption that $e \leqq f$ implies $e f=f e=e$.

The interesting results on a naturally ordered regular semigroup $S$ with a greatest idempotent $u$ have been obtained by Blyth and McFadden in [1] as follows.

(a) Every $x \in S$ has a maximum inverse $x^{\circ}$ in $S$.

(b) The set $S^{\circ}=\left\{x^{\circ}: x \in S\right\}$ of maximum inverses of $S$ forms a multiplicative inverse transversal of $S$, and $S^{\circ}=u S u$.

Let $S$ be a naturally ordered regular semigroup in which each element $x$ has a maximum inverse $x^{\circ}$. The Green's relation $\mathscr{R}$ [resp. $\mathscr{L}$ ] is called regular on $S$ if $x \leqq y$ implies $x x^{\circ} \leqq y y^{\circ}\left[\operatorname{resp} . x^{\circ} x \leqq y^{\circ} y\right]$ for any $x, y \in S$.

A structure theorem on a naturally ordered regular semigroup with maximum inverses has been obtained by Blyth and McFadden ([2, Theorem 6.2]). In this case, there are the assumptions that the set $S^{\circ}$ of maximum inverses is a multiplicative inverse transversal of $S$ and that $\mathscr{R}$ and $\mathscr{L}$ are regular on $S$. In Section 2, we show that if $\mathscr{R}$ and $\mathscr{L}$ are regular on a naturally ordered regular semigroup $S$, then the set $S^{\circ}$ of maximum inverses of $S$ is a multiplicative inverse transversal of $S$.

An idempotent $u$ of a regular semigroup $S$ is called medial if $x=x u x$ for every $x \in\langle E(S)\rangle$, where $\langle E(S)\rangle$ denotes the subsemigroup of $S$ generated by the set $E(S)$ of idempotents of $S$. A medial idempotent $u$ is called normal if $u\langle E(S)\rangle u$ is a semilattice. In [5], McAlister and McFadden have shown that a regular semigroup $S$ with a normal 
medial idempotent $u$ can be naturally ordered in such a way that $u$ is the greatest idempotent of $S$.

In Section 3, we show that if a regular semigroup $S$ has a multiplicative inverse transversal $S^{\circ}$, and if $x^{\circ}$ is the unique inverse of $x \in S$ in $S^{\circ}$, then $S$ can be naturally ordered in such a way that $x^{\circ}$ is the maximum inverse of $x$, and that $\mathscr{R}$ and $\mathscr{L}$ are regular on $S$.

\section{Multiplicative inverse transversals}

Let $S$ be a regular semigroup with an inverse transversal $S^{\circ}$. If $x \in S$, the unique element of $V(x) \cap S^{\circ}$ is denoted by $x^{\circ}$, and $x^{\circ \circ}$ denotes $\left(x^{\circ}\right)^{\circ}$, where $V(x)$ denotes the set of inverses of $x$. A subset $Q$ of $S$ is called a quasi-ideal if $Q S Q \subseteq Q$.

We restate some results about regular semigroups with inverse transversals which will be used in this paper:

Let $S$ be a regular semigroup with an inverse transversal $S^{\circ}$. Then:

$\left(1^{\circ}\right)$ If $S^{\circ}$ is multiplicative, then $S^{\circ}$ is a quasi-ideal of $S$ ([4, Lemma 1.2]).

$\left(2^{\circ}\right)$ If $S^{\circ}$ is a quasi-ideal of $S$, then

(i) $e^{\circ} \in E\left(S^{\circ}\right)$ and $g g^{\circ}=g$ [resp. $\left.f^{\circ} f=f\right]$ imply $e^{\circ} g=e^{\circ} g^{\circ} \quad\left[\right.$ resp. $\left.f e^{\circ}=f^{\circ} e^{\circ}\right]([4$, Lemma 1.6]),

(ii) $x \mathscr{R} y[$ resp. $x \mathscr{L} y]$ implies $x x^{\circ}=y y^{\circ} \quad\left[\right.$ resp. $\left.x^{\circ} x=y^{\circ} y\right]$ for every $x, y \in S([4$, Proposition 1.7]), and

(iii) $a x b=a x^{\circ \circ} b$ for every $a, b \in S^{\circ}$ and for every $x \in S$ ([7, Proposition 1.7]).

Lemma 1.1. Let $S$ be a regular semigroup with an inverse transversal $S^{\circ}$ which is a quasi-ideal of $S$. Suppose that $e^{\circ} \in E\left(S^{\circ}\right)$ for every $e \in E(S)$. If $x^{\prime} \in V(x)$ for any $x \in S$, then $\left(x x^{\prime}\right)^{\circ}=x^{\circ \circ} x^{\circ},\left(x^{\prime} x\right)^{\circ}=x^{\circ} x^{\circ \circ}$ and $\left(x^{\prime}\right)^{\circ}=x^{\circ \circ}$.

Proof. Let $x \in S$ and let $x^{\prime} \in V(x)$. Since $x x^{\prime} \mathscr{R} x, x x^{\prime}\left(x x^{\prime}\right)^{\circ}=x x^{\circ}$. By the assumption, $\left(x x^{\prime}\right)^{\circ} \in E\left(S^{\circ}\right)$, so that $x x^{\circ}\left(x x^{\prime}\right)^{\circ}=x x^{\prime}\left(x x^{\prime}\right)^{\circ}\left(x x^{\prime}\right)^{\circ}=x x^{\prime}\left(x x^{\prime}\right)^{\circ}=x x^{\circ}$ and $\left(x x^{\prime}\right)^{\circ} x x^{\circ}=$ $\left(x x^{\prime}\right)^{\circ} x x^{\prime}\left(x x^{\prime}\right)^{\circ}=\left(x x^{\prime}\right)^{\circ}$ which shows $x x^{\circ} \mathscr{L}\left(x x^{\prime}\right)^{\circ}$. Thus we have $x^{\circ \circ} x^{\circ}=\left(x x^{\circ}\right)^{\circ} x x^{\circ}=$ $\left(x x^{\prime}\right)^{\circ \circ}\left(x x^{\prime}\right)^{\circ}=\left(x x^{\prime}\right)^{\circ}$. Similarly $x^{\circ} x^{\circ \circ}=\left(x^{\prime} x\right)^{\circ}$. By using the above facts, we have

$$
\begin{aligned}
& x^{\circ \circ} x^{\prime} x^{\circ \circ}=x^{\circ \circ} x^{\prime} x x^{\prime} x^{\circ \circ} x^{\circ} x^{\circ \circ}=x^{\circ \circ} x^{\prime} x x^{\prime}\left(x x^{\prime}\right)^{\circ} x^{\circ \circ}=x^{\circ \circ} x^{\prime} x x^{\circ} x^{\circ \circ} \\
& \quad=x^{\circ \circ}\left(x^{\prime} x\right)^{\circ \circ} x^{\circ} x^{\circ \circ}=x^{\circ \circ} x^{\circ} x^{\circ \circ} x^{\circ} x^{\circ \circ}=x^{\circ \circ}
\end{aligned}
$$

and $x^{\prime} x^{\circ \circ} x^{\prime}=x^{\prime} x x^{\prime} x^{\circ \circ} x^{\circ} x^{\circ \circ} x^{\prime}=x^{\prime} x x^{\prime}\left(x x^{\prime}\right)^{\circ} x^{\circ \circ} x^{\prime}=x^{\prime} x x^{\circ} x^{\circ \circ} x^{\prime}=x^{\prime} x\left(x^{\prime} x\right)^{\circ} x^{\prime}=x^{\prime}$. Consequently $\left(x^{\prime}\right)^{\circ}=x^{\circ \circ}$.

If $S^{\circ}$ is multiplicative, for any $e \in E(S), e^{\circ}=e^{\circ} e e^{\circ}=e^{\circ} e e e^{\circ} \in E\left(S^{\circ}\right)$. Thus we obtain:

Corollary 1.2. Let $S$ be a regular semigroup with a multiplicative inverse transversal $S^{\circ}$. If $x^{\prime} \in V(x)$ for $x \in S$, then $\left(x x^{\prime}\right)=x^{\circ \circ} x^{\circ},\left(x^{\prime} x\right)^{\circ}=x^{\circ} x^{\circ \circ}$ and $\left(x^{\prime}\right)^{\circ}=x^{\circ \circ}$. 
Theorem 1.3. Let $S$ be a regular semigroup with an inverse transversal $S^{\circ}$. Then $S^{\circ}$ is multiplicative if and only if $S^{\circ}$ is a quasi-ideal of $S$ and $e^{\circ} \in E\left(S^{\circ}\right)$ for every $e \in E(S)$.

Proof. Suppose that $S^{\circ}$ is a quasi-ideal of $S$ and $e^{\circ} \in E\left(S^{\circ}\right)$ for every $e \in E(S)$. Let $x, y \in S$ and put $e=x x^{\circ}$. and $f=y^{\circ} y$. Then $f e \in S^{\circ} S S^{\circ} \subseteq S^{\circ}$, so that $(f e)^{\circ \circ}=f e$. Since $e(f e)^{\circ} f \in V(f e)$, by Lemma $1.1 f e=(f e)^{\circ \circ}=\left(e(f e)^{\circ} f\right)^{\circ}$. Since $e(f e)^{\circ} f \in E(S)$, by the assumption $\left(e(f e)^{\circ} f\right)^{\circ} \in E\left(S^{\circ}\right)$. Consequently $y^{\circ} y x x^{\circ} \in E\left(S^{\circ}\right)$. Thus $S^{\circ}$ is multiplicative.

From $\left(1^{\circ}\right)$ the converse is true.

\section{Ordered regular semigroups with maximum inverses}

Let $S$ be a partially ordered regular semigroup in which each element has the maximum inverse. If $x \in S$, the maximum element of $V(x)$ is denoted by $x^{\circ}$, and $x^{\circ 0}$ denotes $\left(x^{\circ}\right)^{\circ}$. The set of maximum inverse of $S$ is denoted by $S^{\circ}$, i.e. $S^{\circ}=\left\{a \in S: a=x^{\circ}\right.$ for some $x \in S\}$. The $\mathscr{R}$-class [resp. $\mathscr{L}$-class] containing $x \in S$ is denoted by $R_{x}$ [resp. $L_{x}$ ].

Proposition 2.1. Let $S$ be a partially ordered regular semigroup with maximum inverses. Then:

(1) $e e^{\circ}\left[\right.$ resp. $\left.e^{\circ} e\right]$ is the maximum idempotent of $R_{e}\left[\right.$ resp. $\left.L_{e}\right]$ for every $e \in E(S)$.

(2) $\left(x^{\circ} x\right)^{\circ} x^{\circ}=x^{\circ}\left(x x^{\circ}\right)^{\circ}=x^{\circ}$ for every $x \in S$.

(3) $x x^{\circ}\left[\right.$ resp. $\left.x^{\circ} x\right]$ is the maximum idempotent of $R_{x}$ [resp. $L_{x}$ ] for every $x \in S$.

(4) $x \mathscr{R} y[$ resp. $x \mathscr{L} y]$ implies $x x^{\circ}=y y^{\circ}\left[\right.$ resp. $\left.x^{\circ} x=y^{\circ} y\right]$ for every $x, y \in S$.

(5) $\left(x x^{\circ}\right)^{\circ}=x^{\circ \circ} x^{\circ}$ and $\left(x^{\circ} x\right)^{\circ}=x^{\circ} x^{\circ \circ}$ for every $x, y \in S$.

Proof. (1) Let $e, f \in E(S)$ with $e \mathscr{R} f$. Since $f \in V(e), f \leqq e^{\circ}$. Thus $f=e f \leqq e e^{\circ}$. Since $e \mathscr{R} e e^{\circ}, e e^{\circ}$ is the maximum idempotent of $R_{e}$.

(2) Let $x \in S$. Since $x^{\circ} x \in V\left(x^{\circ} x\right), x^{\circ} x \leqq\left(x^{\circ} x\right)^{\circ}$, so that $x^{\circ} \leqq\left(x^{\circ} x\right)^{\circ} x^{\circ}$. Conversely, since $\left(x^{\circ} x\right)^{\circ} x^{\circ} \in V(x),\left(x^{\circ} x\right)^{\circ} x^{\circ} \leqq x^{\circ}$. Thus $\left(x^{\circ} x\right)^{\circ} x^{\circ}=x^{\circ}$.

(3) Let $x \in S$. Then by (2) $x x^{\circ}\left(x x^{\circ}\right)^{\circ}=x x^{\circ}$. Therefore, by (1) $x x^{\circ}$ is the maximum idempotent of $R_{x x^{\circ}}=R_{x}$.

(4) By (3), this is clear.

(5) Let $x \in S$. Since $x^{\circ} \mathscr{R} x^{\circ} x$, by (3) $x^{\circ} x^{\circ \circ}=x^{\circ} x\left(x^{\circ} x\right)^{\circ}$. By (2) we have $x^{\circ} x\left(x^{\circ} x\right)^{\circ}=\left(x^{\circ} x\right)^{\circ} x^{\circ} x\left(x^{\circ} x\right)^{\circ}=\left(x^{\circ} x\right)^{\circ}$, so that $x^{\circ} x^{\circ \circ}=\left(x^{\circ} x\right)^{\circ}$.

The following fact is very useful.

If $S$ is a naturally ordered semigroup, then $e \leqq f$ for $e, f \in E(S)$ implies $e=e f e([1$, Theorem 1.1]).

Proposition 2.2. Let $S$ be a naturally ordered regular semigroup with maximum inverses. Then:

(1) $e^{\circ o}$ is an inverse of e for every $e \in E(S)$.

(2) $e^{\circ 0}$ is an idempotent for every $e \in E(S)$. 
(3) If $e^{\circ}$ is an idempotent for $e \in E(S)$, then $e^{\circ}=e^{\circ 0}$.

(4) $x^{000}=x^{\circ}$ for every $x \in S$.

Proof. (1) Let $e \in E(S)$. Since $e \in V(e)$ and $e \in V\left(e^{\circ}\right), e \leqq e^{\circ}$ and $e \leqq e^{\circ 0}$, so that $e \leqq e^{\circ} e^{\circ 0}$ and $e \leqq e^{\circ \circ} e^{\circ}$. By the above fact, $e=e e^{\circ} e^{\circ 0} e=e e^{\circ \circ} e^{\circ} e$. Thus we have $e^{00} e e^{00}=e^{\circ 0} e^{\circ} e e^{\circ} e^{\circ 0} e e e^{\circ 0} e^{\circ} e e^{\circ} e^{\circ \circ}=e^{\circ \circ} e^{\circ} e e^{\circ} e^{\circ \circ}=e^{\circ \circ}$ and $e e^{\circ 0} e=e e^{\circ \circ} e^{\circ} e e e^{\circ} e^{\circ \circ} e=e$. Consequently $e^{\circ \circ} \in V(e)$.

(2) By (1), $e e^{\circ \circ} \in V(e)$, so that $e e^{\circ \circ} \leqq e^{\circ}$. Since $e^{\circ} e e^{\circ \circ} e^{\circ}=e^{\circ} e e^{\circ \circ} e e^{\circ} e e^{\circ}=e^{\circ} e e^{\circ}=e^{\circ}$ and $e e^{\circ \circ} e^{\circ} e e^{\circ \circ}=e e^{\circ \circ}$, ee $e^{\circ \circ} \in \mathrm{V}\left(e^{\circ}\right)$, so that $e e^{\circ \circ} \leqq e^{\circ \circ}$. Thus we have $e^{\circ \circ} e^{\circ \circ}=$ $e^{\circ 0} e e^{00} e^{00} \leqq e^{\circ 0} e^{\circ} e^{00}=e^{00}=e^{\circ 0} e e^{\circ 0} \leqq e^{00} e^{\circ 0}$. Consequently $\left(e^{\circ 0}\right)^{2}=e^{\circ 0}$

(3) Since $e^{\circ \circ} \in V(e), e^{\circ 0} \leqq e^{\circ}$. If $e^{\circ}$ is an idempotent, then $e^{\circ} \in V\left(e^{\circ}\right)$, so that $e^{\circ} \leqq e^{\circ \circ}$. Consequently $e^{\circ}=e^{\circ \circ}$.

(4) Let $x \in S$. By (5) of Proposition $2.1,\left(x x^{\circ}\right)^{\circ}=x^{\circ \circ} x^{\circ}$ is an idempotent, so that by (3) $\left(x x^{\circ}\right)^{\circ}=\left(x x^{\circ}\right)^{\circ \circ}$. Thus we have $x^{\circ 0} x^{\circ}=\left(x x^{\circ}\right)^{\circ}=\left(x x^{\circ}\right)^{\circ \circ}=x^{\circ \circ} x^{\circ 0 \circ}$, and similarly $x^{\circ} x^{00}=x^{000} x^{00}$. Consequently $x^{000}=x^{000} x^{00} x^{000}=x^{\circ} x^{00} x^{\circ}=x^{\circ}$.

In the following Lemmas $2.3,2.4$ and $2.5, S$ denotes a naturally ordered regular semigroup with maximum inverses and suppose that $\mathscr{R}$ and $\mathscr{L}$ are regular on $S$.

Lemma 2.3. $V(x) \cap S^{\circ}=\left\{x^{\circ}\right\}$ for every $x \in S$.

Proof. Let $x \in S$ and let $a \in V(x) \cap S^{\circ}$. Then $a=y^{\circ}$ for some $y \in S$. By (4) of Proposition 2.2, $a=y^{\circ}=y^{\circ 0 \circ}=a^{\circ \circ}$. Since $a \in V(x), a \leqq x^{\circ}$. Thus $a a^{\circ} \leqq x^{\circ} x^{\circ \circ}$ since $\mathscr{R}$ is regular on $S$. Conversely, $x \in V(a)$ implies $x \leqq a^{\circ}$, so that $x^{\circ} x \leqq a^{\circ \circ} a^{\circ}=a a^{\circ}$ since $\mathscr{L}$ is regular on $S$. Thus we have $x^{\circ} x^{\circ \circ}=x^{\circ} x x^{\circ} x^{\circ \circ}=x^{\circ} x\left(x^{\circ} x\right)^{\circ} \leqq a a^{\circ}\left(a a^{\circ}\right)^{\circ}=a a^{\circ}$. Consequently $x^{\circ} x^{\circ \circ}=a a^{\circ}$. Similarly $x^{\circ \circ} x^{\circ}=a^{\circ} a$. Therefore $a^{\circ}=a^{\circ} a x a a^{\circ}=x^{\circ \circ} x^{\circ} x x^{\circ} x^{\circ \circ}=x^{\circ \circ}$, so that $a=a^{\circ 0}=x^{000}=x^{\circ}$.

Lemma 2.4. $e^{\circ} \in E\left(S^{\circ}\right)$ for every $e \in E(S)$.

Proof. Let $e \in E(S)$. By Proposition 2.2, $e^{\circ \circ} \in V(e) \cap E\left(S^{\circ}\right)$. Since $e^{\circ} \in V(e) \cap S^{\circ}$, by Lemma $2.3 e^{\circ}=e^{\circ \circ}$. Consequently $e^{\circ}$ is an idempotent of $S^{\circ}$.

Lemma 2.5. $(x y)^{\circ}=\left(x^{\circ} x y\right)^{\circ} x^{\circ}=y^{\circ}\left(x y y^{\circ}\right)^{\circ}$ for every $x, y \in S$.

Proof. Let $x, y \in S$. Then $x y \mathscr{L} x^{\circ} x y$, so that $(x y)^{\circ} x y=\left(x^{\circ} x y\right)^{\circ} x^{\circ} x y$. Since

$$
x y(x y)^{\circ} \mathscr{R} x y\left(x^{\circ} x y\right)^{\circ} x^{\circ}, x y(x y)^{\circ}=x y(x y)^{\circ}\left(x y(x y)^{\circ}\right)^{\circ}=x y\left(x^{\circ} x y\right)^{\circ} x^{\circ}\left(x y\left(x^{\circ} x y\right)^{\circ} x^{\circ}\right)^{\circ} .
$$

Since

$$
x x^{\circ}, x y\left(x^{\circ} x y\right)^{\circ} x^{\circ} \in E(S)
$$

and 


$$
x y\left(x^{\circ} x y\right)^{\circ} x^{\circ}=x x^{\circ} x y\left(x^{\circ} x y\right)^{\circ} x^{\circ}=x y\left(x^{\circ} x y\right)^{\circ} x^{\circ} x x^{\circ}, x y\left(x^{\circ} x y\right)^{\circ} x^{\circ} \leqq x x^{\circ},
$$

so that $x y(x y)^{\circ}=x y\left(x^{\circ} x y\right)^{\circ} x^{\circ}\left(x y\left(x^{\circ} x y\right)^{\circ} x^{\circ}\right)^{\circ} \leqq x x^{\circ}\left(x x^{\circ}\right)^{\circ}=x x^{\circ}$. Thus $x^{\circ} x y(x y)^{\circ} \leqq x^{\circ}$. Since $\left(x^{\circ} x y\right)^{\circ} x^{\circ} \in V(x y), \quad\left(x^{\circ} x y\right)^{\circ} x^{\circ} \leqq(x y)^{\circ}$. Then we have $x y(x y)^{\circ}=x y\left(x^{\circ} x y\right)^{\circ} x^{\circ} x y(x y)^{\circ} \leqq$ $x y\left(x^{\circ} x y\right)^{\circ} x^{\circ} \leqq x y(x y)^{\circ}$. Consequently $x y(x y)^{\circ}=x y\left(x^{\circ} x y\right)^{\circ} x^{\circ}$, so that $(x y)^{\circ}=(x y)^{\circ} x y(x y)^{\circ}=$ $(x y)^{\circ} x y\left(x^{\circ} x y\right)^{\circ} x^{\circ}=\left(x^{\circ} x y\right)^{\circ} x^{\circ} x y\left(x^{\circ} x y\right)^{\circ} x^{\circ}=\left(x^{\circ} x y\right)^{\circ} x^{\circ}$. Similarly we obtain $(x y)^{\circ}=y^{\circ}\left(x y y^{\circ}\right)^{\circ}$.

Theorem 2.6. Let $S$ be a naturally ordered regular semigroup in which every element $x$ has a maximum inverse $x^{\circ}$, and on which $\mathscr{R}$ and $\mathscr{L}$ are regular. Then the set $S^{\circ}=\left\{x^{\circ}: x \in S\right\}$ of maximum inverses of $S$ is a multiplicative inverse transversal of $S$.

Proof. We show that $S^{\circ}$ is a quasi-ideal of $S$. Let $a, b \in S^{\circ}$ and let $x \in S$. Then $a=a^{\circ \circ}$ and $b=b^{\circ \circ}$. By Lemma 2.5, we have $a \times b(a \times b)^{\circ} a a^{\circ}=a \times b\left(a^{\circ} a x b\right)^{\circ} a^{\circ} a a^{\circ}=a \times b\left(a^{\circ} a x b\right)^{\circ} a^{\circ}=$ $a x b(a x b)^{\circ}=a a^{\circ} a x b(a x b)^{\circ}$, so that $a x b(a x b)^{\circ} \leqq a a^{\circ}$. Similarly $(a x b)^{\circ} a x b \leqq b^{\circ} b$. Therefore we have

$$
\begin{aligned}
& (a \times b)^{\circ \circ}(a \times b)^{\circ} a \times b=\left(a \times b(a \times b)^{\circ}\right)^{\circ} a \times b(a \times b)^{\circ} a \times b \leqq\left(a a^{\circ}\right)^{\circ} a a^{\circ} a \times b \\
& =a \times b=a \times b(a x b)^{\circ} a \times b \leqq(a \times b)^{\circ \circ}(a \times b)^{\circ} a x b,
\end{aligned}
$$

consequently $a \times b=(\mathrm{axb})^{\circ \circ}(\mathrm{axb})^{\circ} \mathrm{axb}$. Similarly we obtain $a \times b=a \times b(a \times b)^{\circ}(a \times b)^{\circ \circ}$. Thus we have $a \times b=(a x b)^{\circ \circ}(a x b)^{\circ} a x b(a x b)^{\circ}(a x b)^{\circ \circ}=(a x b)^{\circ \circ} \in S^{\circ}$, which shows $S^{\circ}$ is a quasiideal of $S$. Since $S$ is a regular semigroup, a quasi-ideal of $S$ is a subsemigroup. By Lemma 2.3, $S^{\circ}$ is an inverse transversal. By Lemma 2.4 and Theorem $1.3, S^{\circ}$ is multiplicative.

\section{The ordering on regular semigroups}

Throughout this section $S$ denotes a regular semigroup with a multiplicative inverse transversal $S^{\circ}$. If $x \in S$, the unique element of $V(x) \cap S^{\circ}$ is denoted by $x^{\circ}$, and $x^{\circ \circ}$ denotes $\left(x^{\circ}\right)^{\circ}$. Let $I=\left\{e \in S: e e^{\circ}=e\right\}$ and $\Lambda=\left\{f \in S: f^{\circ} f=f\right\}$. Then, $I$ [resp. $\Lambda$ ] is a left [resp. right] normal band, i.e. ef $g=e g f$ [resp. $e f g=f e g$ ] for every $e, f, g \in I$ [resp. $\Lambda$ ] [4]. The following fact has been obtained by Blyth and McFadden in [2]:

$S$ is algebraically isomorphic to

$$
W=\left\{[e, a, f) \in I \times S^{\circ} \times \Lambda: e^{\circ}=a a^{-1}, f^{\circ}=a^{-1} a\right\},
$$

where multiplication in $W$ is defined by

$$
(e, a, f)(g, b, h)=\left(e a f g a^{-1}, a f g b, b^{-1} f g b h\right) .
$$

We shall define a relation $\leqq$ on $I$ by $e \leqq g$ if and only if $g e=e$ or $g e=e^{\circ}$ for any $e, g \in I$, and similarly on $\Lambda$, using the same symbol $\leqq ; f \leqq h$ if and only if $f h=f$ or $f h=f^{\circ}$ for any $f, h \in \Lambda$. 
Lemma 3.1. The above defined relation on $I[$ resp. $\Lambda]$ is an order relation.

Proof. It is evident that $e \leqq e$ for every $e \in I$.

Let $e, g \in I$ with $e \leqq g$ and $g \leqq e$. The following three cases can be considered:

(1) $g e=e$ and $e g=g$. (2) $g e=e$ and $e g=g^{\circ}$. (3) $g e=e^{\circ}$ and $e g=g^{\circ}$. For (1), since $I$ is left normal, $e=g e=e g e=e e g=e g=g$. For (2), we have $g=g g^{\circ}=g e g=g g e=g e=e$. For (3), we have $e^{\circ} g^{\circ}=e^{\circ} g=e^{\circ} e g=e^{\circ} e^{\circ}=e^{\circ}$ and similarly $e^{\circ} g^{\circ}=g^{\circ} e^{\circ}=g^{\circ}$, so that $e^{\circ}=g^{\circ}$. Thus we have $e=e e^{\circ}=e g e=g^{\circ} e=e^{\circ} e=e^{\circ}$ and similarly $g=g^{\circ}$. Consequently $e=g$.

Let $e, g, m \in I$ with $e \leqq g$ and $g \leqq m$. Then the following four cases can be considered: (1) $g e=e$ and $m g=g$. (2) $g e=e^{\circ}$ and $m g=g$. (3) $g e=e$ and $m g=g^{\circ}$. (4) $g e=e^{\circ}$ and $m g=g^{\circ}$. For each case, we can prove that $e \leqq m$. In each case, the proof is simple but tedious, so the proofs are omitted.

$I$ [resp. $\Lambda$ ] is clearly naturally ordered under the above defined order $\leqq$. Since In $\Lambda=E\left(S^{\circ}\right)$, we have $e^{\circ} \omega f^{\circ}$ in $E(S)$ if and only if $e^{\circ} \leqq f^{\circ}$ in $I$ [resp. $\Lambda$ ] for $e^{\circ}, f^{\circ} \in E\left(S^{\circ}\right)$.

Lemma 3.2. $I(\cdot, \leqq)[$ resp. $\Lambda(\cdot, \leqq)]$ is a naturally ordered left [resp. right] normal band, and $e^{\circ}$ is the maximum inverse of $e \in I$ [resp. $\left.\Lambda\right]$.

Proof. Let $e, g \in I$ with $e \leqq g$ and let $m \in I$. If $g e=e$, then $m g m e=m m g e=m e$ and gmem $=g e m m=e m$, so that $m e \leqq m g$ and $e m \leqq g m$. If $g e=e^{\circ}$, then mgme=mmge= $m e^{\circ}=m e$ and $g m e m=g e m m=e^{\circ} m=e^{\circ} m^{\circ}=(e m)^{\circ}$, so that $m e \leqq m g$ and $e m \leqq g m$. Let $e \in I$ and let $g \in V(e) \cap I$. Then $e^{\circ}=g^{\circ}$, so that $e^{\circ} g=g^{\circ} g=g^{\circ}$. Consequently $g \leqq e^{\circ}$.

Lemma 3.3. Let $e, g \in I$ with $e \leqq g$ and let $f, h \in \Lambda$ with $f \leqq h$. Then fewhg.

Proof. The following four cases can be considered: (1) $g e=e$ and $f h=f$. (2) $g e=e^{\circ}$ and $f h=f$. (3) $g e=e$ and $f h=f^{\circ}$. (4) $g e=e^{\circ}$ and $f h=f^{\circ}$. Since $S^{\circ}$ is multiplicative, $f e, h g \in E\left(S^{\circ}\right)$. Then, for (1), we have $h g f e=f e h g=f h g e h g=f h g e^{\circ} h g=f h g h g e^{\circ}=$ $f h g e=f e$, so that $f e \omega h g$. For each other case, we can similarly prove that fewhg.

It is well-known that an inverse semigroup $S^{\circ}$ can be partially ordered by setting, for any $a, b \in S^{\circ}, a \leqq b$ if and only if $a=e b$ for some $e \in E\left(S^{\circ}\right)$. We use the cartesian ordering on $W=\left\{(e, a, f) \in I \times S^{\circ} \times \Lambda: e^{\circ}=a a^{-1}, f^{\circ}=a^{-1} a\right\}:(e, a, f) \leqq(g, b, h)$ if and only if $e \leqq g$ in $I, a \leqq b$ in $S^{\circ}$ and $f \leqq h$ in $\Lambda$.

Theorem 3.4. Under the cartesian ordering

$$
W=\left\{(e, a, f) \in I \times S^{\circ} \times \Lambda: e^{\circ}=a a^{-1}, f^{\circ}=a^{-1} a\right\}
$$

is a naturally ordered regular semigroup in which each element $(e, a, f)$ has the maximum inverse $\left(f^{\circ}, a^{-1}, e^{\circ}\right)$, and on which $\mathscr{R}$ and $\mathscr{L}$ are regular.

Proof. Let $(e, a, f),(g, b, h) \in W$ with $(e, a, f) \leqq(g, b, h)$ and let $(m, c, n) \in W$. Then $e \leqq g$, $a \leqq b$ and $f \leqq h$, so that $a^{-1} \leqq b^{-1}$ and by Lemma $3.3 \mathrm{fm} \leqq h m$ in $S^{\circ}$. Therefore af $m c \leqq b h m c$, af $m a^{-1} \leqq b h m b^{-1}$ and $c^{-1} f m c \leqq c^{-1} h m c$ in $S^{\circ}$, so that af $m a^{-1} \leqq b h m b^{-1}$ in $I$ and $c^{-1} f m c \leqq c^{-1} h m c$ in $\Lambda$. Thus eaf $m a^{-1} \leqq g b h m b^{-1}$ in $I$ and $c^{-1} f m c n \leqq c^{-1} h m c n$ 
in $\Lambda$. Consequently $(e, a, f)(m, c, n) \leqq(g, b, h)(m, c, n)$. Similarly we can show $(m, c, n)(e, a, f) \leqq(m, c, n)(g, b, h)$.

Let $(e, a, f),(g, b, h) \in E(W) \quad$ with $\quad(e, a, f)(g, b, h)=(g, b, h)(e, a, f)=(e, a, f) . \quad$ Then $g b h e b^{-1}=e, a f g b=a$ and $b^{-1} f g b h=f$. Thus $g e=e$ and $f h=f$, so that $e \leqq g$ and $f \leqq h$. By Theorem 1.3, $a, b \in E\left(S^{\circ}\right)$. Therefore $b a=a b=a f g b b=a f g b=a$, so that $a \leqq b$. Consequently $W$ is naturally ordered.

Let $(e, a, f) \in W$ and let $(g, b, h) \in V((e, a, f))$. By Corollary $1.2, b=a^{-1}$, so that $g \leqq g^{\circ}=b b^{-1}=a^{-1} a=f^{\circ}$ and similarly $h \leqq e^{\circ}$. Consequently $(g, b, h) \leqq\left(f^{\circ}, a^{-1}, e^{\circ}\right)=$ $(e, a, f)^{\circ}$. Thus each element $(e, a, f) \in W$ has the maximum inverse $\left(f^{\circ}, a^{-1}, e^{\circ}\right)$.

Let $(e, a, f),(g, b, h) \in W$ with $(e, a, f) \leqq(g, b, h)$. Then $a \leqq b$, so that $a a^{-1} \leqq b b^{-1}$. Since $(e, a, f)(e, a, f)^{\circ}=\left(e, a a^{-1}, a a^{-1}\right)$ and $(g, b, h)(e, b, h)^{\circ}=\left(g, b b^{-1}, b b^{-1}\right), \quad(e, a, f)(e, a, f)^{\circ} \leqq$ $(g, b, h)(g, b, h)^{\circ}$. Thus $\mathscr{R}$ is regular on $W$. Similarly $\mathscr{L}$ is regular on $W$.

We shall define a relation on $S$ by, for any $x, y \in S, x \leqq y$ if and only if $x x^{\circ} \leqq y y^{\circ}$ in $I$, $x^{\circ \circ} \leqq y^{\circ \circ}$ in $S^{\circ}$ and $x^{\circ} x \leqq y^{\circ} y$ in $\Lambda$. Then $x \leqq y$ in $S$ implies $\left(x x^{\circ}, x^{\circ \circ}, x^{\circ} x\right) \leqq\left(y y^{\circ}, y^{\circ \circ}, y^{\circ} y\right)$ in $W$. Conversely, $(e, a, f) \leqq(g, b, h)$ in $W$ implies eaf $(e a f)^{\circ}=e \leqq g=g b h(g b h)^{\circ}$ in $I$, $(e a f)^{\circ \circ}=a \leqq b=(g b h)^{\circ \circ}$ in $S^{\circ}$ and $(e a f)^{\circ} e a f=f \leqq h=(g b h)^{\circ} g b h$ in $\Lambda$, so that $e a f \leqq g b h$ in $S$. Thus the isomorphism $\theta: W \rightarrow S$ defined by $(e, a, f) \theta=e a f$ is isotone.

Thus we obtain:

Theorem 3.5. Let $S$ be a regular semigroup with a multiplicative inverse transversal $S^{\circ}$, and let $V(x) \cap S^{\circ}=\left\{x^{\circ}\right\}$ for every $x \in S$. Then $S$ can be naturally ordered in such a way that $x^{\circ}$ is the maximum inverse of any element $x$ of $S$ and that $\mathscr{R}$ and $\mathscr{L}$ are regular on $S$.

\section{REFERENCES}

1. T. S. BLYTH and R. B. MCFadden, Naturally ordered regular semigroups with a greatest idempotent. Proc. Roy. Soc. Edinburgh 91A (1981), 107-122.

2. T. S. BLYTH and R. B. MCFAdDEN, Regular semigroups with a multiplicative inverse transversal, Proc. Roy. Soc. Edinburgh 92A (1982), 253-270.

3. T. S. BLYTh and R. B. McFadDEN, On the construction of a class of regular semigroups, $J$. Algebra 81 (1983), 1-22.

4. D. B. MCAlister and R. B. McFadDeN, Regular semigroups with inverse transversals, Quart. J. Math. Oxford (2) 34 (1983), 459-474.

5. D. B. MCAlister and R. B. MCFADDEN, Maximum idempotents in naturally ordered regular semigroups, Proc. Edinburgh Math. Soc. 26 (1983), 213-220.

6. TATSUHiкo SAIto, Construction of a class of regular semigroups with an inverse transversal, Proc. Conference on Theory and Application of Semigroups, at Greifswald (GDR) (1984), 108-113.

7. TAтsuHiko Salto, Relationship between the inverse transversals of a regular semigroup, Semigroup Forum 33 (1986), 245-250.

SHIMONOSEKI UNiversity of Fisheries

Yoshimi, ShimonosekI

JAPAN 